\title{
ROLES OF ROBOTS IN HUMAN SOCIETY: CHALLENGES AND CASE STUDIES
}

Kerstin Dautenhahn

Adaptive Systems Research Group

Univerisity of Hertfordshire, Department of Computer Science, UK

My talk will discuss different possible roles of robots in human societies, focussing on studies that we carried out in the Aurora project (http://www.aurora-project.com/). Challenges identified, and lessons learnt from studying interactions between robots and children with autism will be presented in the broader context of service robotics, with a particular focus on "a robot in my house" scenario which is central to the Cogniron project. 